\title{
Inhibiting Glycosaminoglycan Chain Polymerization Decreases the Inhibitory Activity of Astrocyte-Derived Chondroitin Sulfate Proteoglycans
}

\author{
Tracy L. Laabs, ${ }^{1,2}$ Hang Wang, ${ }^{1}$ Yasuhiro Katagiri, ${ }^{1}$ Thomas McCann, ${ }^{1}$ James W. Fawcett, ${ }^{2}$ and Herbert M. Geller ${ }^{1}$ \\ ${ }^{1}$ Developmental Neurobiology Section, National Heart, Lung, and Blood Institute, National Institutes of Health, Bethesda, Maryland 20892, and ${ }^{2}$ Cambridge \\ University Centre for Brain Repair, Cambridge CB2 2PY, United Kingdom
}

\begin{abstract}
Chondroitin sulfate proteoglycans (CSPGs) are upregulated in the CNS after injury and participate in the inhibition of axon regeneration mainly through their glycosaminoglycan (GAG) side chains. In the present study, we have identified a new way to alleviate the inhibition of axonal regeneration by CSPG GAGs. We have successfully decreased the amount of CSPG GAG produced by astrocytes by targeting chondroitin polymerizing factor (ChPF), a key enzyme in the CSPG biosynthetic pathway. Using short interfering RNA (siRNA), we reduced ChPF mRNA levels by $70 \%$ in both the Neu7 astrocyte cell line and primary rat astrocytes. This reduction leads to a decrease in ChPF protein levels and a reduced amount of CSPG GAG chains in the conditioned media (CM) of these cells. Secretion of neurocan by primary astrocytes and NG2 core protein by Neu7 cells transfected with ChPF siRNA is not decreased, suggesting that inhibiting GAG chain synthesis does not affect core protein trafficking from these cells. CM from siRNA-treated Neu7 cells is a less repulsive substrate for axons than CM from control cells. In addition, axonal outgrowth from cerebellar granule neurons is increased on or in CM from ChPF siRNA-treated Neu7 cells. These data indicate that targeting the biosynthesis of CSPG GAG is a potentially new therapeutic avenue for decreasing CSPG GAG produced by astrocytes after CNS injury.
\end{abstract}

Key words: axon guidance; granule neuron; siRNA; chondroitin polymerizing factor; Neu7 cells; matrix

\section{Introduction}

Chondroitin sulfate proteoglycans (CSPGs) are a family of extracellular matrix (ECM) molecules with diverse functions in development (Bandtlow and Zimmermann, 2000; Schwartz and Domowicz, 2004), infection (Uyama et al., 2006), inflammation (Taylor and Gallo, 2006), and tissue morphogenesis and cell division (Mizuguchi et al., 2003). Of these, the role of CSPGs in mammalian CNS injury and plasticity has been the subject of intense investigation (Carulli et al., 2005). The production of CSPGs by astrocytes is rapidly upregulated after CNS injury, and their deposition in the ECM forms a major component of the glial scar that serves to prevent axon regeneration (McKeon et al., 1991; Gates et al., 1996; Davies et al., 1997, 1999; Fitch and Silver, 1997). In vitro, CSPGs alone are inhibitory to neurite extension and can present a boundary to growing axons (Snow et al., 1990).

CSPGs have two major structural features: a single protein core and one or more unbranched, polysaccharide glycosaminoglycan (GAG) chains. Removal of CSPG GAG chains with the enzyme chondroitinase $\mathrm{ABC}(\mathrm{cABC})$ reduces the inhibitory

\footnotetext{
Received Dec. 21, 2006; revised Nov. 14, 2007; accepted Nov. 21, 2007.

This work was supported by the Intramural Research Program of the National Heart, Lung, and Blood Institute, National Institutes of Health.

Correspondence should be addressed to Dr. Herbert M. Geller, Developmental Neurobiology Section, National Heart, Lung, and Blood Institute, National Institutes of Health, 10 Center Drive, MSC 1754, Bethesda, MD 20892 1754. E-mail: geller@helix.nih.gov.

DOI:10.1523/JNEUROSCI.2807-07.2007

Copyright $\odot 2007$ Society for Neuroscience $\quad 0270-6474 / 07 / 2714494-08 \$ 15.00 / 0$
}

properties of CSPGs on neurite growth and guidance in vitro (Snow et al., 1990), and reduces the boundary forming activity of the Neu7 astrocyte cell line (Powell et al., 1997). In vivo, cABC treatment increases axonal regeneration and, in some cases, functional recovery after CNS injury (McKeon et al., 1995; Moon et al., 2001; Bradbury et al., 2002). This suggests that astrocytederived CSPGs exert their inhibitory effects through their GAG chains.

There are several major disadvantages to the use of $\mathrm{CABC}$ as a treatment option for spinal cord injury in humans. Because CSPGs are continuously being synthesized after injury, a single injection may not prevent the eventual reestablishment of the inhibitory CNS matrix. This is further compounded by its potentially short-lived enzymatic activity (Tester et al., 2007) and the fact that $\mathrm{CABC}$ digestion leaves behind "stubs," which may have biological activity (Lemons et al., 2003). Finally, because cABC is a natural product of the bacterium Proteus vulgaris, repeated injections are likely to produce an immune reaction. An alternate approach to this problem may be through the use of an agent that continually reduces the polymerization of GAG chains on CSPG core proteins.

The biosynthesis of CSPGs is the result of multiple enzymes that serve to add sugar moieties to a serine residue of the core protein (Fig. 1). It begins with the addition of xylose, followed by two galactose molecules and a glucuronic acid (GlcA), completing the core-protein-GAG tetrasaccharide linkage region common to all sulfated proteoglycans. The chain is then polymerized 

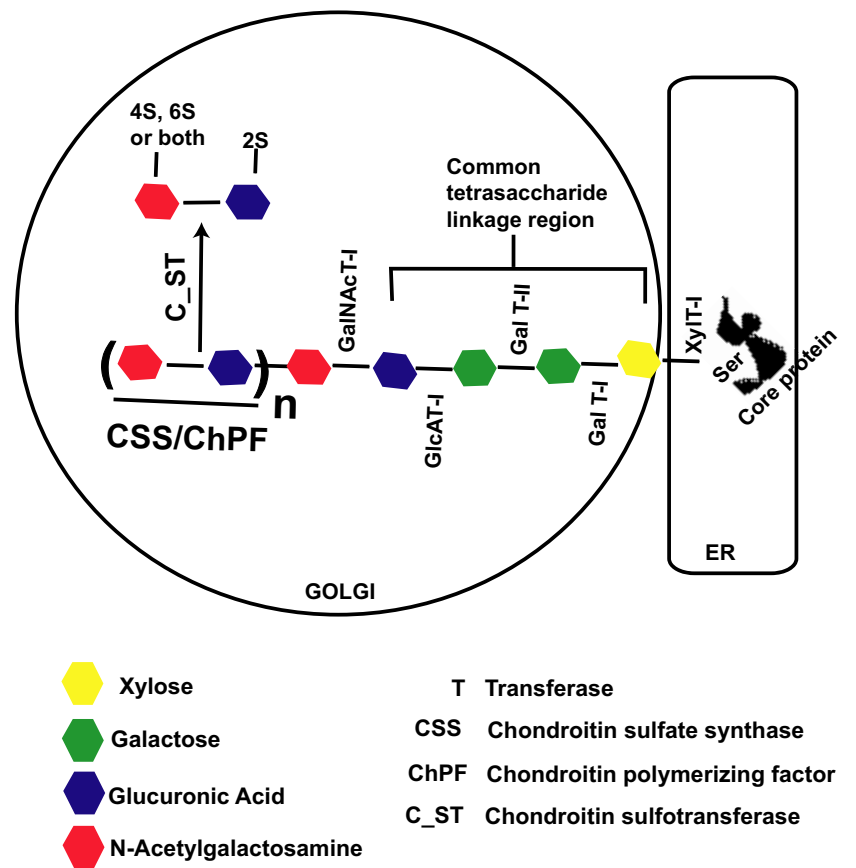

Figure 1. Biosynthesis of CS. GAG chain formation is initiated on a serine residue of the core protein. It begins in the endoplasmic reticulum (ER) with the addition of xylose (Xyl) by xylosyltransferase-I. Subsequently, in the Golgi, two galactose (Gal) moieties are added by galactosyltransferase-I and -II, respectively. The addition of the first GlcA residue by glucuronyltransferase-I completes the common tetrasaccharide linkage region. The transfer of a GalNAc by $N$-acetylgalactosaminyl transferase-I commits the GAG chain to being a galactosaminoglycan (CS/dermatan sulfate) rather than a glucosaminoglycan (heparan sulfate). The chain is polymerized by the addition of repeating GICA and GalNAc through the activity of the chondroitin synthase family of enzymes (CSS) and ChPF. The chain is then modified by chondroitin sulfotransferases, which put a sulfate moiety (S) on the 4 or 6 position of the GalNAc or the 2 position of the GlcA.

by the addition of repeating disaccharide units consisting of $N$-acetyl galactosamine (GalNAc) and GlcA. This is followed by further modification by sulfation and epimerization. Polymerization occurs through the action of an enzyme complex consisting of chondroitin synthase (ChSy) (Kitagawa et al., 2001) and chondroitin polymerizing factor (ChPF) (Kitagawa et al., 2003). These enzymes are broadly distributed in tissues, including brain. In this study, we sought to determine whether we could make ChPF the rate-limiting enzyme for CSPG GAG chain biosynthesis in astrocytes by decreasing expression levels using a plasmidbased short interfering RNA (siRNA). We also investigated whether decreasing the amount of ChPF protein in astrocytes would change the composition, as well as the biological activity, of the extracellular matrix made by these cells.

\section{Materials and Methods}

Cell culture. Glial cell cultures were prepared from the brains of newborn Sprague Dawley rats [postnatal day 1 (P1) to P2] as previously described (Petroski et al., 1991). Briefly, the cerebral cortices were dissected out, freed of meninges, and collected in HBSS (Invitrogen, Carlsbad, CA). They were minced with sterile scissors and digested in $0.1 \%$ trypsin and $0.02 \%$ DNase (Invitrogen) for $20 \mathrm{~min}$ at $37^{\circ} \mathrm{C}$. Cells were triturated, passed through a $70 \mu \mathrm{m}$ filter, and washed two times with culture media. The cells were seeded in $75 \mathrm{~cm}^{2}$ flasks and cultured in DMEM containing 10\% fetal bovine serum (FBS) and 1\% penicillin-streptomycin (P-S; Invitrogen). After reaching confluency, the cultures were shaken to remove macrophages and progenitor cells. The adherent astrocytes were removed from the flask and counted, and the required number of cells were used for transfection.
The Neu7 cell line (Fok-Seang et al., 1995) was grown in DMEM containing $10 \%$ FBS and 1\% P-S. Cultures of cerebellar granule neurons (CGNs) were prepared as previously described (Levi et al., 1984). Briefly, cerebella were removed from postnatal 5-8 d C56BL/6 mice and collected in HBSS, dissociated with trypsin and DNase, and passed through a $40 \mu \mathrm{m}$ cell strainer. After dissociation, cells were resuspended in Neurobasal-A media supplemented with B27, $2 \mathrm{~mm}$ glutamine, $25 \mathrm{~mm} \mathrm{KCl}$, and 1\% P-S (CGN media). Cells were seeded on coverslips for the boundary crossing spot assay at a density of 70,000 cells/well.

siRNA and transfection. siRNA target sequences were designed using a combination of the Tuschl rules and rational siRNA design. The IMG800 (pSuppressorNeo, Imgenex, San Diego, CA) vector was used for construction of short hairpin RNA (shRNA) for rat ChPF, GenBank NM_001005906. For construction, two complementary oligonucleotides containing three different rat ChPF sequences were synthesized (Qiagen, Valencia, CA) and annealed to generate double-stranded DNAs, which were cloned into the SalI and $\mathrm{XbaI}$ cloning sites of IMG-800. Clones were sequenced using the primer $5^{\prime}$-AATACGTGACGTAGAAAGTA- $3^{\prime}$ to ensure the correct insert. A GFP vector (pEGFP-N1, Clontech, Mountain View, CA) was used in combination with the siRNA construct to determine transfection efficiency. The negative control plasmid contains a scrambled sequence that does not show significant homology to rat, mouse, or human sequences. Only one of the three sequences against ChPF that was cloned into IMG-800 was effective at decreasing mRNA levels. The sense sequence is $5^{\prime}$-aagctgccgtaatgacatcgt- $3^{\prime}$. To confirm that the gene silencing of ChPF by our siRNA in IMG- 800 could be achieved by other siRNA constructs with a different target region, we also used Dharmacon ON-TARGETplus SMARTpool chemically synthesized siRNA (Dharmacon, Lafayette, CO). The control in this case was a siRNA pool also purchased from Dharmacon. In both cases, Neu7 cells were transfected using the Amaxa nucleofection technology (Amaxa, Gaithersburg, MD). Neu7 cells were grown to confluency, trypsinized, spun down, and resuspended in solution from the rat astrocyte nucleofector kit following the Amaxa guidelines for cell line transfection. After transfection, cells were cultured in media containing serum for the stated number of days before isolating RNA, or the serum-containing media was replaced with serum-free media after $24 \mathrm{~h}$, and the cells were cultured in serum-free media for the stated number of days before collecting conditioned media and cells for SDS-PAGE.

RNA extraction and quantitative PCR. RNA was extracted using the Absolutely RNA miniprep kit following the manufacturer's protocol (Stratagene, La Jolla, CA). Genomic DNA was removed by DNase I treatment. RNA was reverse transcribed using SuperScript III reverse transcriptase (Invitrogen) and subjected to quantitative PCR (qPCR). Quantitative PCR was performed in a Chromo4 (Bio-Rad, Hercules, CA) with DyNAmo HS SYBR Green kit (New England Biolabs, Ipswich, MA). PCR conditions consisted of a $15 \mathrm{~min}$ hot start at $95^{\circ} \mathrm{C}$ followed by 45 cycles of the following sequence: $94^{\circ} \mathrm{C}$ for $15 \mathrm{~s}, 58^{\circ} \mathrm{C}$ for $15 \mathrm{~s}$, and $72^{\circ} \mathrm{C}$ for $25 \mathrm{~s}$. The primer sequences are as follows: ChPF forward, $5^{\prime}$-TGCCTGATGCCACCTACAC-3'; ChPF reverse, 5' ${ }^{\prime}$-TAGTCTCTCCACCGATGAAGTC-3'; glyceraldehyde-3-phosphate dehydrogenase (GAPDH) forward, 5'-AAGGTGGTGAAGCAGGCATC TG-3'; and GAPDH reverse, 5' -TGGGTGGTCCAGGGTTTCTT AC-3'. All samples were run in triplicate, and all mRNA levels were normalized to the level of GAPDH. All PCR fragments were purified using a gel extraction kit (Qiagen), and sequenced. cDNAs for ChPF and GAPDH were used as a template for PCR to obtain a standard curve.

SDS-PAGE and Western blotting. Conditioned media (CM) was collected, concentrated in a centricon YM-30 (Millipore, Billerica, MA) in the presence of protease inhibitors (Cocktail Set V; Calbiochem, La Jolla, $\mathrm{CA}$ ), and then subjected to SDS-PAGE using a $6 \%$ polyacrylamide gel (Invitrogen). To compare the amount of secreted proteins in the CM, equal volumes of the original conditioned media were loaded. The proteins were then transferred to a polyvinylidene difluoride (PVDF) membrane (Millipore) and blocked for $1 \mathrm{~h}$ in $5 \%$ milk. The blots were then incubated with monoclonal antibody CS-56 (Sigma, St. Louis, MO), which recognizes the glycosaminoglycan portion of native CSPG, rabbit polyclonal anti-NG2 (Millipore), which recognizes both the intact NG2 
proteoglycan and the core protein, or monoclonal anti-neurocan (MAB5212, Millipore).

Cells were collected in SDS sample buffer plus protease inhibitors, and protein concentration was determined using a BCA protein assay kit (Pierce, Rockford, IL) with BSA as a standard. Equal amounts of protein were subjected to SDS-PAGE using a $10 \%$ polyacrylamide gel (Invitrogen). $\beta$-Actin was used as a loading control for cell lysates.

For immunoblotting of ChPF, we generated a custom rabbit antipeptide antibody (Open Biosystems, Huntsville, AL) against the peptide sequence RLSEDLYHRCRQSVLEG, amino acids 734-750 of rat chondroitin polymerizing factor (GenBank NP_001005906). We also expressed a V5-epitope tagged full-length $\mathrm{ChPF}$ protein by cloning the rat ChPF cDNA into $\mathrm{pEF}$ 6/V5-His (Invitrogen). The following primers were used: forward, 5'-TAGGATCCGCCATGCGGGCGTCGCTG-3'; reverse, 5'-ATGCGGCCGCGCCATAGGTGCTGTTACCCT-3'; and the cDNA was obtained using rat astrocyte RNA as a template. pEF6ChPF-V5 was transfected into $293 \mathrm{~T}$ cells using Mammalian $\mathrm{CaPO}_{4}$ transfection kit (Stratagene), and cell lysates were prepared and used as a positive control for immunoblotting experiments. HRP-conjugated secondary antibodies were used and signals were visualized using SuperSignal West Dura (Pierce).

ELISA. Relative amounts of chondroitin sulfate (CS) were measured by ELISA. Briefly, 96-well microtiter plates (Immulon 4; Dynex Technologies, Chantilly, VA) pretreated with poly-L-lysine (PLL) were coated with CM from Neu7 cells or astrocytes treated with a control or siRNA against ChPF. After blocking, primary antibodies against chondroitin sulfate (CS-56) or chondroitin-6-sulfate (MAB2035; Millipore) were incubated, followed by incubation with anti-mouse secondary antibody $\mathrm{F}\left(\mathrm{ab}^{\prime}\right) 2$ fragment conjugated with HRP (anti-IgM, Abcam, Cambridge, MA; anti-IgG, Amersham Biosciences, Pittsburgh, PA). Binding was measured with a microplate reader (Multiskan MCC/340; Labsystems, Helsinki, Finland) using SureBlue TMB Microwell Peroxidase Substrate (KPL, Gaithersburg, MD). For antibody MAB2035, CM samples were digested with $10 \mathrm{mU}$ cABC (Seikagaku, Tokyo, Japan) for $1 \mathrm{~h}$ at $37^{\circ} \mathrm{C}$ to expose the antigen.

Axon growth of CGNs in the presence of Neu7 CM or with Neu7 CM as a substrate. CM from cells transfected with a control or siRNA against $\mathrm{ChPF}$ was collected after $72 \mathrm{~h}$. For use as a growth medium, CM was mixed with an equal volume of DMEM and CGN media supplemented with B27 and CGNs were cultured in the mix on a PLL- and laminincoated surface. For use as a substrate, CM was mixed with $0.1 \mu \mathrm{g} / \mathrm{ml}$ laminin in DMEM and allowed to immobilize on coverslips at $37^{\circ} \mathrm{C}$ for $4 \mathrm{~h}$. The solution was removed and CGNs were cultured on the coverslips for $48 \mathrm{~h}$ in CGN media. After $48 \mathrm{~h}$, cells were fixed and stained for $\beta$-III tubulin (Sigma). Images were obtained from randomly selected fields and analyzed for neurite length using Image (available at http://rsb.info.nih.gov/ij/).

Axon guidance spot assay. An axon guidance assay was performed as previously described (Meiners et al., 1999). Briefly, an interface between PLL and CM was created by placing a $5 \mu \mathrm{l}$ drop of concentrated conditioned medium alone $(\mathrm{CM})(50 \% \mathrm{CM})$ or with cABC treatment $(\mathrm{CM}$ digested overnight at $37^{\circ} \mathrm{C}$ ), or the appropriate percentage of $\mathrm{CM}$ in HBSS together with Texas Red. The mixture was placed in the center of a glass PLL-coated coverslip and allowed to immobilize in a humidified chamber for $2 \mathrm{~h}$ at $37^{\circ} \mathrm{C}$. Texas Red was used to visualize the interface, and Texas Red alone was used as a control. CGNs were then plated on the coverslip and cultured for $48 \mathrm{~h}$ before fixation and staining with an antibody directed against $\beta$-III tubulin (Sigma). The number of axons crossing the spot was divided by the total number of axons within 10 $\mu \mathrm{m}$ of the spot to give the percentage of crossing. Only axons growing toward the immobilized protein were counted, and no axons whose soma was at the interface were counted. The percentage crossing for each experimental condition was divided by the percentage crossing onto Texas Red alone to normalize to control. Each experiment was performed three times, and statistical significance was determined by Student's $t$ test.
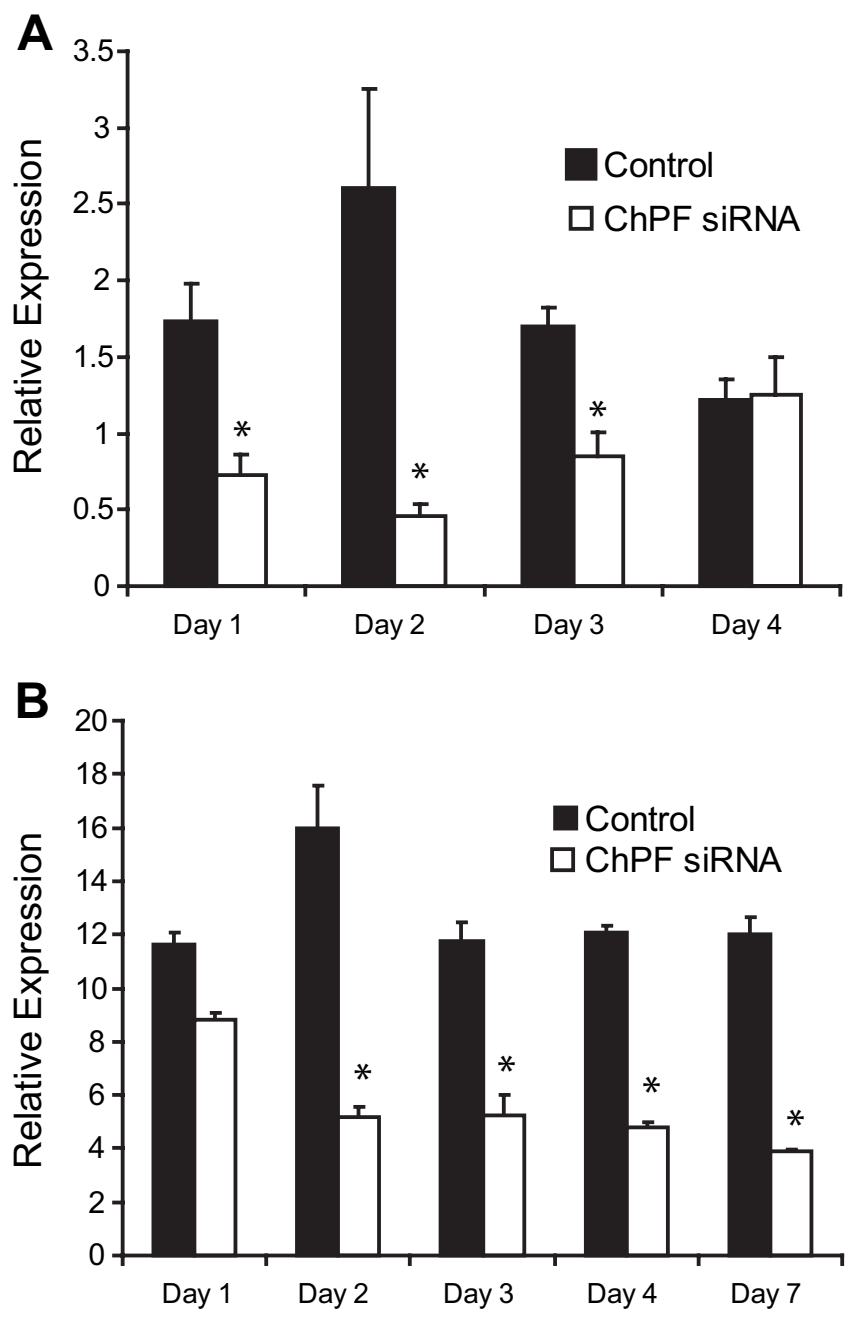

Figure 2. siRNA reduces ChPF mRNA levels in a time-dependent manner. $\boldsymbol{A}$, Neu7 cells were transfected with a control or a plasmid containing siRNA against ChPF. RNA was extracted at 1 , 2,3 , or $4 \mathrm{~d}$ posttransfection (dpt), reverse transcribed, and subjected to qPCR. The amount of ChPF transcript was converted to copy number and normalized to GAPDH. ChPF mRNA levels were lowest at $2 \mathrm{~d}$ after transfection and returned to control levels by $4 \mathrm{~d}$. $\boldsymbol{B}$, Primary rat astrocytes were transfected with a control or a plasmid containing shRNA against ChPF, and RNA was collected at the indicated dpt. ChPF mRNA levels were significantly decreased by $2 \mathrm{~d}$ after transfection and remained down for at least $7 \mathrm{~d}$. ${ }^{*} p<0.05$ by ANOVA with post hoc comparisons. Error bars indicate SEM.

\section{Results}

ChPF mRNA levels are decreased in astrocytes after treatment with siRNA against $\mathrm{ChPF}$

siRNA is an effective way of silencing genes and has recently been used for gene therapy applications (for review, see Shuey et al., 2002). Here, we use a plasmid-based siRNA system for the knockdown of ChPF in Neu7 cells. Neu7 cells are a rapidly dividing cell line that produces an extracellular matrix that contains a large quantity of CSPGs; both Neu7 cells and their extracellular matrix are inhibitory to neurite outgrowth (Fok-Seang et al., 1995). Because plasmid-based siRNA is transient, we first determined the time course of mRNA reduction by an siRNA, selected from a panel of three, in these cells. In Neu7 cells transfected with ChPF siRNA, ChPF mRNA levels were significantly reduced as early as $24 \mathrm{~h}$ after transfection and remained decreased for $3 \mathrm{~d}$ but returned to control-transfected cell levels by $4 \mathrm{~d}$ after transfection (Fig. 2). Transfection efficiency is consistently $75-80 \%$ for Neu7 cells with electroporation. An increase in relative levels of ChPF 
A

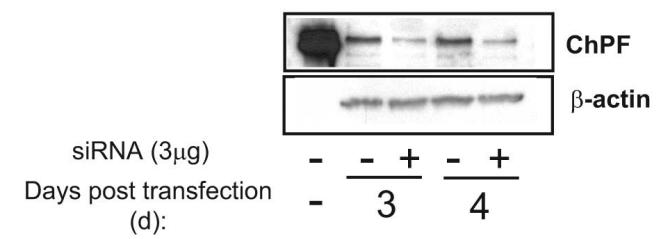

B
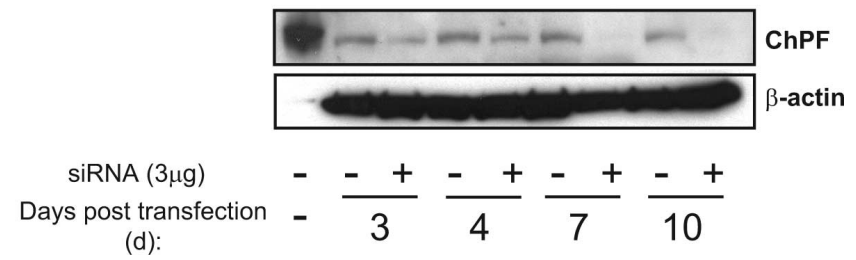

Figure 3. siRNA against ChPF decreases protein levels in astrocytes. Cells were grown to confluency, trypsinized, and transfected using electroporation with either a control vector or a vector containing an siRNA against ChPF. For protein extraction, cells were grown in serumcontaining media for $1 \mathrm{~d}$ to allow attachment after transfection. The media was changed to serum free after $1 \mathrm{~d}$, and the cells were collected after $2 \mathrm{~d}$ [ $3 \mathrm{~d}$ posttransfection (dpt)], $3 \mathrm{~d}$ ( $4 \mathrm{dpt}$ ), or, in the case of astrocytes, $6 \mathrm{~d}(7 \mathrm{dpt})$ or $9 \mathrm{~d}(10 \mathrm{dpt})$. Cell lysates were prepared with SDS sample buffer, and equal amounts of total protein were loaded in a $10 \%$ gel. Protein was transferred to PVDF membrane and blotted for ChPF using our custom made anti-peptide antibody. Blots were washed thoroughly, reblocked, and probed for $\beta$-actin to determine equal loading. $\boldsymbol{A}$, Neu7 cell protein expression after transfection. $\boldsymbol{B}$, Primary rat astrocyte protein expression after transfection.

mRNA in control-transfected cells was observed $2 \mathrm{~d}$ after transfection, most likely caused by recovery from plating after electroporation. We were unable to assay for the effects of the siRNA beyond $4 \mathrm{~d}$ in Neu7 cells because they divide rapidly and the monolayer loses attachment to the culture surface. We obtained equivalent results using Dharmacon ON-TARGETplus SMARTpool ChPF siRNA against ChPF to ensure that we were not producing off-target effects from our plasmid-based system (supplemental Fig. 1, available at www.jneurosci.org as supplemental material). We saw no change in phenotype or significant difference in cell number between the control- and siRNA-transfected cells with either transfection reagent.

Activated astrocytes are the main cell type present in the glial scar. Both in vitro and in vivo, activated astrocytes express neurite outgrowth-inhibitory CSPGs (McKeon et al., 1999; Asher et al., 2000; Davies et al., 2004). This makes them an appropriate target for CSPG biosynthesis inhibitors. They also form stable monolayers, allowing the effects of the siRNA to be assayed over a longer period than is possible in Neu7 cells. We sought to determine whether our siRNA was effective at decreasing ChPF mRNA levels in primary rat astrocytes. Transfection efficiency for primary astrocytes is also $75-80 \%$ consistently with electroporation. Consistent with the fact that primary cells divide more slowly than Neu7 cells and exhibit contact inhibition, ChPF mRNA levels remained lower than control levels for at least $7 \mathrm{~d}$ after transfection (Fig. 2).

\section{ChPF siRNA reduces ChPF protein levels}

We hypothesized that the suppression of ChPF mRNA in siRNAtransfected cells would lead to decreased ChPF protein levels in these cells. To test this hypothesis, we transfected Neu7 cells with a control or siRNA against $\mathrm{ChPF}$ and collected cells at various time points. Cells were collected for RNA extraction and qPCR after $2 \mathrm{~d}$ to evaluate ChPF mRNA levels (Fig. 3A). Cells were collected for protein extraction 3 or $4 \mathrm{~d}$ after transfection to assess ChPF protein levels. Cell lysates were analyzed by SDS-PAGE and Western blotting using a custom made rabbit anti-peptide ChPF antibody. A full-length V5-epitope-tagged ChPF was expressed in $293 \mathrm{~T}$ cells as a positive control. Consistent with a $60-70 \%$ decrease in ChPF mRNA levels at $2 \mathrm{~d}$ (data not shown), we observed a $60 \%$ reduction in $\mathrm{ChPF}$ protein levels by $3 \mathrm{~d}$ after transfection. ChPF protein levels in siRNA-transfected Neu7 cells were lower than in control-transfected cells, and this difference was sustained at least until $4 \mathrm{~d}$ after transfection (Fig. 3A).

We then investigated whether ChPF protein levels were similarly decreased by siRNA in primary rat astrocytes. After transfection with siRNA, primary astrocytes also showed a consistent $60-70 \%$ decrease in ChPF mRNA levels compared with cells transfected with a control vector (data not shown). Primary astrocytes were collected for protein extraction at 3, 4, 7, and $10 \mathrm{~d}$ after transfection. ChPF protein levels in ChPF siRNAtransfected astrocytes were lower than controls by $3 \mathrm{~d}$ after transfection and remained lower than the control cell levels for as long as $10 \mathrm{~d}$ after transfection (Fig. $3 B$ ).

\section{ChPF siRNA reduces GAG chain production by astrocytes}

We first tested whether the decrease in ChPF enzyme levels in cells transfected with ChPF siRNA would result in a decrease in glycanated CSPGs in the media. To determine this, we collected and concentrated serum-free media from Neu7 cells or primary astrocytes transfected with a control or ChPF siRNA. We probed for GAG chains using CS-56 in either Western blot or ELISA assays (data not shown) (Fig. 4). CS-56 recognizes sulfated epitopes on intact CSPGs (Ito et al., 2005). Both assays revealed a significant decrease in GAG chains in the media of the ChPF siRNA-transfected cells compared with control-transfected cells. The Western blot showed a decrease in GAG as early as $3 \mathrm{~d}$ after transfection in Neu7 cells, which indicates that the turnover of CSPG is fairly rapid in the Neu7 cell line (Fig. 4A). The ELISA with primary astrocytes showed a significant decrease in CS-56 reactivity as well (data not shown). To ensure that the decrease in GAG chains in the media was not attributable to a lower number of cells surviving transfection, we trypsinized and counted the cells after collection of the conditioned media. There was no significant difference in the number of cells between the controland siRNA-transfected groups (data not shown). An equivalent reduction in GAG chain synthesis was observed using the SMARTpool ChPF siRNA (supplemental Fig. 1, available at www.jneurosci.org as supplemental material), confirming that the effects were not attributable to off-target actions of our shRNA.

Because there is a potential that decreasing sugar chain biosynthesis could alter the trafficking of CSPG core proteins, we assessed core protein production from Neu7 cells by two outputs. First, we performed a Western blot probing for NG2 core protein, because it is the major axon growth-inhibitory CSPG secreted from Neu7 cells (Fidler et al., 1999) (Fig. 4B). We detected no significant change in NG2 core protein in the media, indicating that the trafficking of the NG2 core protein is not altered after inhibiting glycosylation. We also performed an ELISA on CM using MAB2035, an antibody that recognizes the "stub" tetrasaccharide linkage region left behind on a CSPG after digestion with cABC. The stub detected by MAB2035 was also not significantly decreased in ChPF siRNA-treated cells (data not shown). In primary astrocytes, we evaluated the production of neurocan, one of the major proteoglycans secreted by these cells. Neurocan levels in $\mathrm{CM}$ were not significantly changed after treatment of primary astrocytes with ChPF siRNA (Fig. 4C). These results indicate that 
A

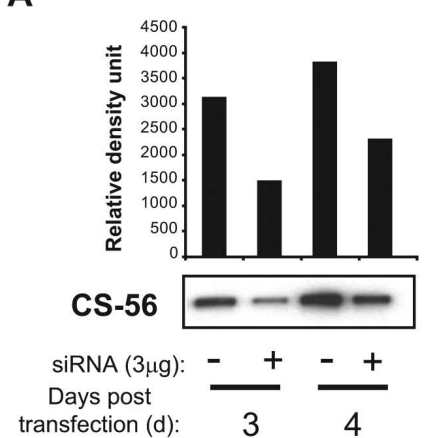

C

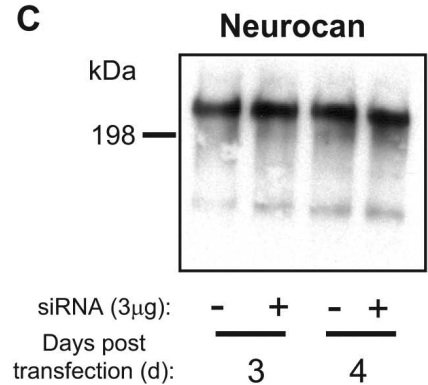

Figure 4. CS-56 reactivity but not core protein is reduced in siRNA-transfected astrocyte CM. Cells were grown to confluency, trypsinized, and transfected with a control or siRNA against ChPF. After $1 \mathrm{~d}$, the media was changed to serum free and collected 2 (3d) or 3 (4d) days later and concentrated, and equal volumes of the original conditioned media were run on a $6 \%$ gel. $A$, CS- 56 reactivity decreases in cells treated with shRNA against ChPF by $3 \mathrm{~d}$ and remains down at $4 \mathrm{~d}$ after transfection. $\boldsymbol{B}, \mathrm{NG} 2$ core protein reactivity in the conditioned media of Neu7 cells remains the same whether the cells are transfected with a control or siRNA against ChPF. C, Neurocan reactivity in the conditioned media of primary astrocytes is unchanged in primary astrocytes transfected with ChPF siRNA.

siRNA treatment selectively altered glycanation of CSPG core proteins while not altering their secretion from the cells.

siRNA-treated Neu7 cell conditioned media is a less repulsive substrate for axons

Primary astrocyte monolayers are generally permissive to neurite outgrowth (Noble et al., 1984; Fallon, 1985), whereas Neu7 cells and their matrix are inhibitory substrates for neurite outgrowth (Fidler et al., 1999). Elimination of CSPG GAG chains with cABC reduces the inhibitory effects of Neu7 cells (Powell and Geller, 1999), and we found the same reduction in inhibition after treatment of Neu7 CM with cABC. With this in mind, Neu7 cells were used as a model system to test the hypothesis that interrupting polymerization of CSPG GAG chains could alleviate CSPGmediated inhibition of axon growth.

We hypothesized that the reduced GAG on CSPGs in conditioned media from siRNA-treated Neu7 cells would be a less inhibitory substrate compared with control-transfected Neu7 conditioned media. To examine this, we tested the ability of conditioned media from siRNA-treated Neu7 cells to repel axons in an axon guidance assay (Meiners et al., 1999). This assay quantifies the number of axons that either turn or cross at a boundary between PLL and a protein of interest. A spot of CM from control-transfected or siRNA-transfected cells was immobilized on a PLL-coated coverslip. CGNs were cultured for $48 \mathrm{~h}$, and the behavior of their axons was analyzed at the PLL/CM interface. Representative images of CGN behavior at a CM border are shown in Figure 5. Whereas only 33\% of axons crossed at a
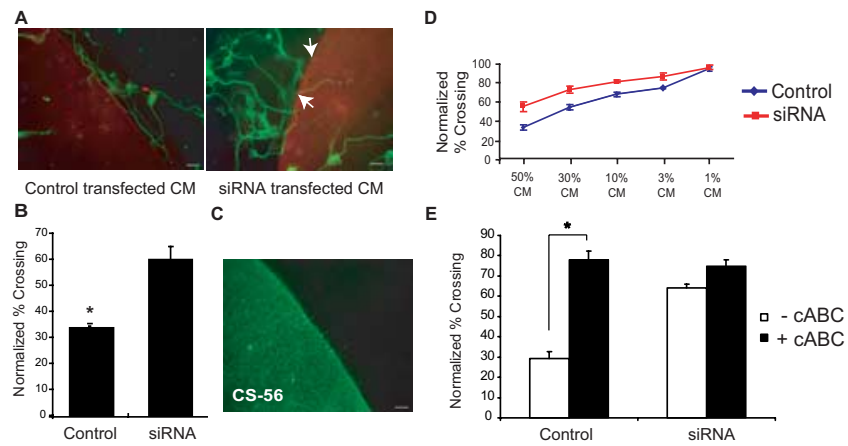

Figure 5. Treatment of Neu7 cells with siRNA against $\mathrm{ChPF}$ renders the conditioned media more permissive to axon growth. $A, B, D$, Neu7 cells were grown to confluency, trypsinized, and transfected with a control or siRNA against ChPF. After $1 \mathrm{~d}$, the media was changed to serumfree media, and the cells were cultured in serum-free media for $48 \mathrm{~h}$. After $48 \mathrm{~h}$, the media was collected, concentrated, and a percentage was mixed with Texas Red dye, dropped onto a PLL-coated glass coverslip, and allowed to immobilize. Cerebellar granule neurons were then plated and cultured for $48 \mathrm{~h}$ before fixation and staining. $A$, Representative pictures of a spot of control-transfected Neu7 CM and siRNA-transfected CM. The siRNA-transfected CM was more likely to support axons crossing the boundary (arrows). Scale bar, $10 \mu \mathrm{m}$. B, Bar graph of the difference in crossing between the highest concentration of $C M$ in control or siRNA-treated Neu7 cells. C, Representative picture of a spot stained with CS-56. CSPG concentration was not increased around the border. Scale bar, $10 \mu \mathrm{m}$. D, Dose-response of axon crossing at a border with indicated percentage of conditioned media. $E$, Comparison of siRNA treatment with chondroitinase $(C A B C)$ treatment.

boundary of control CM and PLL, $60 \%$ of axons crossed at a border of siRNA-treated CM and PLL. This indicates that siRNA transfection decreased the inhibitory activity of the CSPGs present in the CM. Although one study has shown that the concentration of CSPG at a spot border is consistently higher if the spot is allowed to dry (Tom et al., 2004), we found that the concentration of CSPG on the spot is uniform if the spot is incubated in a humidified chamber (Fig. 5C). Staining of the spot with anti-NG2 antibody demonstrated that the ability of the CSPG core protein NG2 to adhere to the culture surface was not altered by decreased glycanation (data not shown). A dose-response of crossing was also observed in CM from both control and ChPF siRNA-transfected cells (Fig. 5D). As the percentage of CM in the spot was decreased, increased crossing was observed. Finally, we compared siRNA treatment with chondroitinase treatment. CM from control or siRNA-transfected cells was divided into two aliquots, and one was digested with chondroitinase overnight at $37^{\circ} \mathrm{C}$. Spot assays were performed with the four groups of $\mathrm{CM}$ (Fig. $5 E$ ). Only $30 \%$ of axons crossed onto a spot of Neu7 CM, whereas $65 \%$ of axons crossed onto CM from siRNA-treated Neu7 cells. cABC treatment resulted in $70 \%$ of axons crossing, which was not significantly different from crossing achieved with siRNA treatment.

\section{Neurite outgrowth from CGNs is increased on or in CM from} ChPF siRNA-treated Neu7 cells

The effects of extracellular matrix molecules on axon growth and axon guidance have been shown to differ (Powell et al., 1997). With this in mind, we investigated the ability of ChPF siRNAtransfected Neu7 cell CM to support axon outgrowth in two ways. First, CM was used as a substrate for CGN growth. PLLand laminin-coated coverslips were coated with either control or siRNA-transfected CM. Neurons were plated on the substrate, and their growth was evaluated $48 \mathrm{~h}$ later. Neurite outgrowth on $\mathrm{CM}$ from control-transfected cells was significantly less than outgrowth on CM from siRNA-treated Neu7 cells (Fig. 6A,C). 


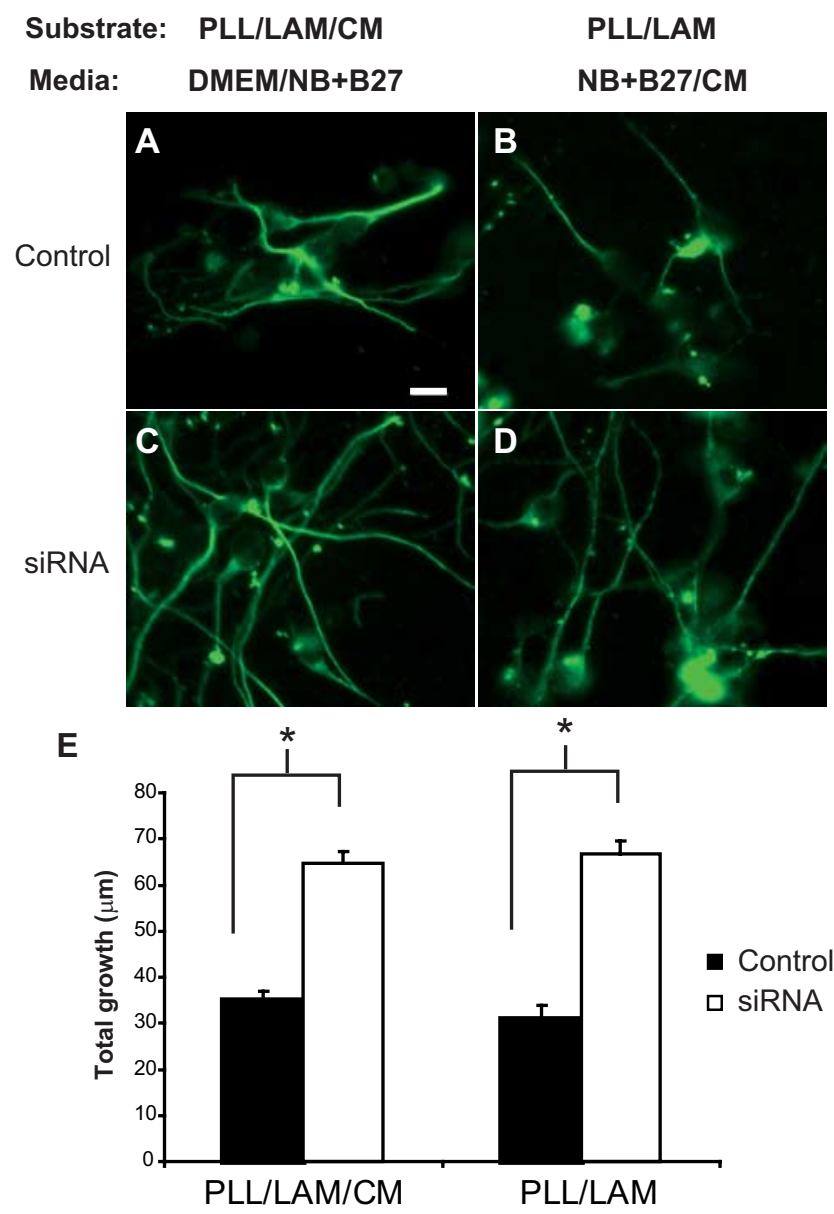

Figure 6. Increased neurite outgrowth on or in CM from ChPF siRNA-treated Neu7 cells. Neu7 cells were transfected with a control plasmid or siRNA against ChPF and grown in serumcontaining media for $1 \mathrm{~d}$. The media was changed to serum free, and the cells were grown for 3 additional days, at which time the media was collected. Cerebellar granule neurons were either grown on a combination of PLL, laminin (LAM), and immobilized CM $(\boldsymbol{A}, \boldsymbol{C})$ in half DMEM and half Neurobasal (NB) + B27 or on a combination of PLL and LAM in half NB + B27 and halfCM from the Neu7 cells $(\boldsymbol{B}, \boldsymbol{D})$. $\boldsymbol{E}$, Quantification. $n=70$ for each group. ${ }^{*} p<0.001$. Scale bar, 10 $\mu \mathrm{m}$.

We also tested the effect of CM in solution in the medium on neurite outgrowth. CGNs were grown on PLL and laminincoated coverslips in the presence of control or siRNA-transfected cell CM. Axons of CGNs grown on a combination of PLL/LAM in CM from siRNA-treated Neu7 cells grew significantly better than CGNs grown on the same substrate in control CM (Fig. $6 B, D$ ). In both cases, CGN axon length was doubled in siRNA-treated cell $\mathrm{CM}$ compared with control (Fig. 6E). These data indicate that treatment of Neu7 cells with ChPF siRNA decreases the amount of inhibitory GAG chains present in secreted matrix.

\section{Discussion}

The GAG chain moieties of CSPGs have major actions on axonal growth and guidance. Eliminating these GAG chains with cABC has become a favored approach to promoting recovery of function after CNS injury. We demonstrate here a feasible alternative to $\mathrm{CABC}$, which is reducing the polymerization of CSPG GAG chains by targeting a key biosynthetic enzyme, ChPF. We have demonstrated the efficacy of this approach using both primary astrocytes and the axon growth-inhibitory astrocytic cell line, Neu7, whose inhibitory properties are attributable to the secretion of CSPG GAGs into their extracellular matrix. Treating as- trocytes with siRNA directed against ChPF reduced their production of glycanated CSPGs. This reduction in secreted GAG did not affect secretion of core proteins, indicating that siRNA achieved a selective reduction of GAG chain synthesis.

The fact that inhibition of CSPG GAG chain synthesis in cells increases the ability of their matrix to support neurite outgrowth is not entirely unexpected. CSPG GAG chains have been implicated in a variety of barrier-forming phenomena, including cell division (Mizuguchi et al., 2003), neural cell migration (Kubota et al., 1999; Perissinotto et al., 2000; Perris and Perissinotto, 2000), and inhibition of axon regeneration (Silver and Miller, 2004). cABC is effective at removal of GAG chains at the lesion site in vivo (McKeon et al., 1995; Moon et al., 2001; Bradbury et al., 2002), distal to the lesion site, when combined with a peripheral nerve graft (Houle et al., 2006), as well as in vitro. We show that the biological effects of inhibiting ChPF in vitro are not significantly different from using $\mathrm{CABC}$, and we predict that such actions in vivo will similarly result in increased growth.

The reduction in GAG-decorated CSPGs in Neu7 CM reduced the ability of the CM to form a boundary to growing axons. In parallel, the GAG-reduced CM was also less of an inhibitory substrate to axonal outgrowth. Neu7 cells are a cell line with demonstrated neurite outgrowth inhibitory properties (FokSeang et al., 1995), in contrast to primary astrocytes, which are highly permissive in vitro (Noble et al., 1984). Neu7 cells rapidly divide and do not form an indefinitely stable monolayer, preventing coculture from being performed in these experiments. Moreover, we are interested in the matrix produced by these cells and did not want to confound the results with the milieu of cell surface molecules as well as bound factors present in a coculture situation. NG2 has been identified as the major component of Neu7 matrix, and several studies, including our own, have suggested that much of the inhibitory activity of NG2 resides in the core protein (Fidler et al., 1999). However, certain domains of NG2 require GAG chain attachment for their inhibitory activity (Ughrin et al., 2003). Moreover, although NG2 is the major proteoglycan produced by Neu7 cells, it is not the only inhibitory proteoglycan produced. Neu7 cells also produce versican and potentially other unknown glycanated proteoglycans (Fidler et al., 1999). Additionally, both CS GAG chains and NG2 core protein are responsible for the inhibitory properties of Neu7 cells, but the two do not necessarily need to be attached to each other. The fact that GAG chain synthesis inhibition did not completely eliminate the inhibitory activity of Neu7 medium is consistent with a role for the NG2 core protein as well as the GAG chains in inhibiting axonal growth.

The rate of turnover of CSPGs in the nervous system is largely unknown. CSPG synthesis is rapidly upregulated after CNS injury, and CSPG core proteins digested by CABC can persist for weeks (Bruckner et al., 1998). The turnover rate may be extremely variable because there are many CSPG core proteins present after a lesion (Morgenstern et al., 2002), and each of these could have a different rate of synthesis and/or degradation. Therefore, a single dose of $\mathrm{CABC}$, although effective at eliminating existing GAG chains, will not prevent their resynthesis. Thus, if the rate of synthesis is more rapid than the rate of elimination or if cABC is rapidly inactivated, GAG chains will be reinserted into the glial scar ECM. Regenerating axons that have insufficient time to traverse the lesion area will then encounter CSPGs, which limit their growth. This problem can be addressed by targeting the polymerization of the CSPG GAG. In the presence of a ChPF enzyme inhibitor, CSPGs decorated with GAG chains would not 
be synthesized, no matter what the turnover rate of the core protein.

The formation of CSPGs is catalyzed by three major classes of enzymes: (1) xylosyltransferase (XT) is responsible for initiating CS GAG chain synthesis on the serine residue of the core protein; (2) the CSS/ChPF family of enzymes catalyzes polymerization of GAG chains by incorporating GlcA and GalNAc; and (3) a family of chondroitin sulfotransferases catalyze the transfer of sulfate from 3 ' -phosphoadenosine 5 ' -phosphosulfate to the appropriate position on the GAG sugar. Each of these processes is then a potential target for therapeutic intervention. Targeting GAG chain initiation by reducing XT has been shown to decrease CS GAG chains in the glial scar and increase regeneration of microtransplanted dorsal root ganglion cells through a stab lesion in an adult rat (Grimpe and Silver, 2004). However, this region is common among other sulfated proteoglycans, such as heparan sulfate proteoglycans (HSPGs), and, thus, not specific for CSPGs. HSPGs have been shown to bind growth factors permissive for dopaminergic neuron sprouting after a nigrostriatal lesion in an adult rat (Moon et al., 2002), and HSPG-binding proteins can promote axon growth (Kinnunen et al., 1996), so decreasing their presence at the lesion site may be unfavorable. In contrast, several CSPGs are upregulated and inhibitory to neurite outgrowth after a lesion (McKeon et al., 1991; Fitch and Silver, 1997; Davies et al., 1999), so specifically decreasing GAG chain synthesis on all CSPGs could enhance the growth of axons through a lesion.

One potential drawback of targeting the biosynthetic pathway of CSPGs is the fact that some of the enzymes involved in the synthesis and polymerization of CS GAG chains have many isoforms, and multiple activities that could potentially compensate for the lack of one enzyme. However, ChPF was found to be necessary for polymerization along with ChSy (Kitagawa et al., 2003). After transfection of astrocytes with ChPF siRNA, we observe an $\sim 50 \%$ decrease in GAG chain immunoreactivity. Because $\sim 75 \%$ of cells were transfected, this implies a two-thirds reduction in the transfected cells. In our various axon growth assays, this led to a doubling in boundary crossing and in neurite length on the more permissive substrate. Although only one isoform of ChPF has been identified, there may be other enzymes that have polymerase activity. If so, a multiple RNAi approach targeting a sequence common to multiple isoforms, as well as a transfection method that increases efficiency to near $100 \%$, may further decrease the output of CS-GAGs, resulting in enhanced neuronal growth.

Gene knock-down strategies are being proposed for many different therapeutic applications in nervous system diseases (Lundstrom, 2007). The major issues being confronted are ensuring that the appropriate cells are targeted, that the duration of the effect is sufficient to promote recovery, and that the delivery system does not provoke an immune reaction. Systemic application of siRNA is not favorable because of the rapid degradation of nucleic acids in vivo and, in the case of preventing CS synthesis, may cause arthritis in rapidly turning over cartilage. Viral vectors have been developed that effectively express shRNA in brain tissue (Xia et al., 2004). Alternately, sustained release of siRNA can be achieved by conjugating the nucleic acids to biodegradable microparticles (Khan et al., 2004), which may be a viable option for CNS disorders.

Because the turnover of CSPGs in the CNS matrix is probably slow, the optimum treatment will probably be a dual therapy that involves initial treatment with $\mathrm{cABC}$ to eliminate GAGs already present at the injury site, along with a treatment such as siRNA that decreases GAG synthesis, thereby preventing the reappear- ance of inhibition. Recent experiments have shown that a single injection of $\mathrm{cABC}$ can prevent a rise in GAG for up to a week (Lin et al., 2007), so the blockade of synthesis can be delayed for several days after injury. The other major effect of CSPGs in the CNS is in the control of plasticity, and removal of GAGs with cABC can enhance plasticity in the adult CNS (Pizzorusso et al., 2002; Rhodes and Fawcett, 2004). Reducing GAG synthesis may be an alternative method of producing longer-term increases in plasticity, which could be helpful in a range of CNS disorders.

Inhibitory CSPGs are only one component of the axon growth-inhibitory properties of the CNS. We envision a therapeutic approach that has several different components, perhaps combining proteoglycan synthesis inhibitors with trophic factors that promote axonal sprouting and blockers of myelin inhibition. It is almost certain that a strategy targeting multiple aspects of spinal cord injury will be needed to optimize the recovery process.

\section{References}

Asher RA, Morgenstern DA, Fidler PS, Adcock KH, Oohira A, Braistead JE, Levine JM, Margolis RU, Rogers JH, Fawcett JW (2000) Neurocan is upregulated in injured brain and in cytokine-treated astrocytes. J Neurosci 20:2427-2438.

Bandtlow CE, Zimmermann DR (2000) Proteoglycans in the developing brain: new conceptual insights for old proteins. Physiol Rev 80:1267-1290.

Bradbury EJ, Moon LD, Popat RJ, King VR, Bennett GS, Patel PN, Fawcett JW, McMahon SB (2002) Chondroitinase ABC promotes functional recovery after spinal cord injury. Nature 416:636-640.

Bruckner G, Bringmann A, Hartig W, Koppe G, Delpech B, Brauer K (1998) Acute and long-lasting changes in extracellular-matrix chondroitinsulphate proteoglycans induced by injection of chondroitinase $\mathrm{ABC}$ in the adult rat brain. Exp Brain Res 121:300-310.

Carulli D, Laabs T, Geller HM, Fawcett JW (2005) Chondroitin sulfate proteoglycans in neural development and regeneration. Curr Opin Neurobiol 15:116-120.

Davies JE, Tang X, Denning JW, Archibald SJ, Davies SJ (2004) Decorin suppresses neurocan, brevican, phosphacan and NG2 expression and promotes axon growth across adult rat spinal cord injuries. Eur J Neurosci 19:1226-1242.

Davies SJ, Fitch MT, Memberg SP, Hall AK, Raisman G, Silver J (1997) Regeneration of adult axons in white matter tracts of the central nervous system. Nature 390:680-683.

Davies SJ, Goucher DR, Doller C, Silver J (1999) Robust regeneration of adult sensory axons in degenerating white matter of the adult rat spinal cord. J Neurosci 19:5810-5822.

Fallon JR (1985) Preferential outgrowth of central nervous system neurites on astrocytes and schwann cells as compared with nonglial cells in vitro. J Cell Biol 100:198-207.

Fidler PS, Schuette K, Asher RA, Dobbertin A, Thornton SR, Calle-Patino Y, Muir E, Levine JM, Geller HM, Rogers JH, Faissner A, Fawcett JW (1999) Comparing astrocytic cell lines that are inhibitory or permissive for axon growth: the major axon-inhibitory proteoglycan is NG2. J Neurosci 19:8778-8788.

Fitch MT, Silver J (1997) Glial cell extracellular matrix: boundaries for axon growth in development and regeneration. Cell Tissue Res 290:379-384.

Fok-Seang J, Smith-Thomas LC, Meiners S, Muir E, Du JS, Housden E, Johnson AR, Faissner A, Geller HM, Keynes. RJ, Rogers JH, Fawcett JW (1995) An analysis of astrocytic cell lines with different abilities to promote axon growth. Brain Res 689:207-223.

Gates MA, Fillmore H, Steindler DA (1996) Chondroitin sulfate proteoglycan and tenascin in the wounded adult mouse neostriatum in vitro: dopamine neuron attachment and process outgrowth. J Neurosci 16:8005-8018.

Grimpe B, Silver J (2004) A novel DNA enzyme reduces glycosaminoglycan chains in the glial scar and allows microtransplanted dorsal root ganglia axons to regenerate beyond lesions in the spinal cord. J Neurosci 24:1393-1397.

Houle JD, Tom VJ, Mayes D, Wagoner G, Phillips N, Silver J (2006) Combining an autologous peripheral nervous system "bridge" and matrix 
modification by chondroitinase allows robust, functional regeneration beyond a hemisection lesion of the adult rat spinal cord. J Neurosci 26:7405-7415.

Ito Y, Hikino M, Yajima Y, Mikami T, Sirko S, von Holst A, Faissner A, Fukui S, Sugahara K (2005) Structural characterization of the epitopes of the monoclonal antibodies 473HD, CS-56, and MO-225 specific for chondroitin sulfate D-type using the oligosaccharide library. Glycobiology 15:593-603.

Khan A, Benboubetra M, Sayyed PZ, Ng KW, Fox S, Beck G, Benter IF, Akhtar S (2004) Sustained polymeric delivery of gene silencing antisense ODNs, siRNA, DNAzymes and ribozymes: in vitro and in vivo studies. J Drug Target 12:393-404.

Kinnunen T, Raulo E, Nolo R, Maccarana M, Lindahl U, Rauvala H (1996) Neurite outgrowth in brain neurons induced by heparin-binding growthassociated molecule (HB-GAM) depends on the specific interaction of HB-GAM with heparan sulfate at the cell surface. J Biol Chem 271:2243-2248.

Kitagawa H, Uyama T, Sugahara K (2001) Molecular cloning and expression of a human chondroitin synthase. J Biol Chem 276:38721-38726.

Kitagawa H, Izumikawa T, Uyama T, Sugahara K (2003) Molecular cloning of a chondroitin polymerizing factor that cooperates with chondroitin synthase for chondroitin polymerization. J Biol Chem 278:23666-23671.

Kubota Y, Morita T, Kusakabe M, Sakakura T, Ito K (1999) Spatial and temporal changes in chondroitin sulfate distribution in the sclerotome play an essential role in the formation of migration patterns of mouse neural crest cells. Dev Dyn 214:55-65.

Lemons ML, Sandy JD, Anderson DK, Howland DR (2003) Intact aggrecan and chondroitin sulfate-depleted aggrecan core glycoprotein inhibit axon growth in the adult rat spinal cord. Exp Neurol 184:981-990.

Levi G, Aloisi F, Ciotti MT, Gallo V (1984) Autoradiographic localization and depolarization-induced release of acidic amino acids in differentiating cerebellar granule cell cultures. Brain Res 290:77-86.

Lin R, Kwok JC, Crespo D, Fawcett JW (2007) Chondroitinase ABC has a long-lasting effect on chondroitin sulphate glycosaminoglycan content in the injured rat brain. J Neurochem, in press.

Lundstrom K (2007) Prospects of treating neurological diseases by gene therapy. Curr Opin Investig Drugs 8:34-40.

McKeon RJ, Schreiber RC, Rudge JS, Silver J (1991) Reduction of neurite outgrowth in a model of glial scarring following CNS injury is correlated with the expression of inhibitory molecules on reactive astrocytes. J Neurosci 11:3398-3411.

McKeon RJ, Hoke A, Silver J (1995) Injury-induced proteoglycans inhibit the potential for laminin-mediated axon growth on astrocytic scars. Exp Neurol 136:32-43.

McKeon RJ, Jurynec MJ, Buck CR (1999) The chondroitin sulfate proteoglycans neurocan and phosphacan are expressed by reactive astrocytes in the chronic CNS glial scar. J Neurosci 19:10778-10788.

Meiners S, Mercado ML, Kamal MS, Geller HM (1999) Tenascin-C contains domains that independently regulate neurite outgrowth and neurite guidance. J Neurosci 19:8443-8453.

Mizuguchi S, Uyama T, Kitagawa H, Nomura KH, Dejima K, Gengyo-Ando K, Mitani S, Sugahara K, Nomura K (2003) Chondroitin proteoglycans are involved in cell division of Caenorhabditis elegans. Nature 423:443-448.

Moon LD, Asher RA, Rhodes KE, Fawcett JW (2001) Regeneration of CNS axons back to their target following treatment of adult rat brain with chondroitinase ABC. Nat Neurosci 4:465-466.
Moon LD, Asher RA, Rhodes KE, Fawcett JW (2002) Relationship between sprouting axons, proteoglycans and glial cells following unilateral nigrostriatal axotomy in the adult rat. Neuroscience 109:101-117.

Morgenstern DA, Asher RA, Fawcett JW (2002) Chondroitin sulphate proteoglycans in the CNS injury response. Prog Brain Res 137:313-332.

Noble M, Fok-Seang J, Cohen J (1984) Glia are a unique substrate for the in vitro growth of central nervous system neurons. J Neurosci 4:1892-1903.

Perissinotto D, Iacopetti P, Bellina I, Doliana R, Colombatti A, Pettway Z, Bronner-Fraser M, Shinomura T, Kimata K, Morgelin M, Lofberg J, Perris $\mathrm{R}$ (2000) Avian neural crest cell migration is diversely regulated by the two major hyaluronan-binding proteoglycans PG-M/versican and aggrecan. Development 127:2823-2842.

Perris R, Perissinotto D (2000) Role of the extracellular matrix during neural crest cell migration. Mech Dev 95:3-21.

Petroski RE, Grierson JP, Choi-Kwon S, Geller HM (1991) Basic fibroblast growth factor regulates the ability of astrocytes to support hypothalamic neuronal survival in vitro. Dev Biol 147:1-13.

Pizzorusso T, Medini P, Berardi N, Chierzi S, Fawcett JW, Maffei L (2002) Reactivation of ocular dominance plasticity in the adult visual cortex. Science 298:1248-1251.

Powell EM, Geller HM (1999) Dissection of astrocyte-mediated cues in neuronal guidance and process extension. Glia 26:73-83.

Powell EM, Fawcett JW, Geller HM (1997) Proteoglycans provide neurite guidance at an astrocyte boundary. Mol Cell Neurosci 10:27-42.

Rhodes KE, Fawcett JW (2004) Chondroitin sulphate proteoglycans: preventing plasticity or protecting the CNS? J Anat 204:33-48.

Schwartz NB, Domowicz M (2004) Proteoglycans in brain development. Glycoconj J 21:329-341.

Shuey DJ, McCallus DE, Giordano T (2002) RNAi: gene-silencing in therapeutic intervention. Drug Discov Today 7:1040-1046.

Silver J, Miller JH (2004) Regeneration beyond the glial scar. Nat Rev Neurosci 5:146-156.

Snow DM, Lemmon V, Carrino DA, Caplan AI, Silver J (1990) Sulfated proteoglycans in astroglial barriers inhibit neurite outgrowth in vitro. Exp Neurol 109:111-130.

Taylor KR, Gallo RL (2006) Glycosaminoglycans and their proteoglycans: host-associated molecular patterns for initiation and modulation of inflammation. FASEB J 20:9-22.

Tester NJ, Plaas AH, Howland DR (2007) Effect of body temperature on chondroitinase $\mathrm{ABC}$ 's ability to cleave chondroitin sulfate glycosaminoglycans. J Neurosci Res 85:1110-1118.

Tom VJ, Steinmetz MP, Miller JH, Doller CM, Silver J (2004) Studies on the development and behavior of the dystrophic growth cone, the hallmark of regeneration failure, in an in vitro model of the glial scar and after spinal cord injury. J Neurosci 24:6531-6539.

Ughrin YM, Chen ZJ, Levine JM (2003) Multiple regions of the NG2 proteoglycan inhibit neurite growth and induce growth cone collapse. J Neurosci 23:175-186.

Uyama T, Ishida M, Izumikawa T, Trybala E, Tufaro F, Bergstrom T, Sugahara K, Kitagawa H (2006) Chondroitin 4-O-sulfotransferase-1 regulates $\mathrm{E}$ disaccharide expression of chondroitin sulfate required for herpes simplex virus infectivity. J Biol Chem 281:38668-38674.

Xia H, Mao Q, Eliason SL, Harper SQ, Martins IH, Orr HT, Paulson HL, Yang L, Kotin RM, Davidson BL (2004) RNAi suppresses polyglutamineinduced neurodegeneration in a model of spinocerebellar ataxia. Nat Med $10: 816-820$. 